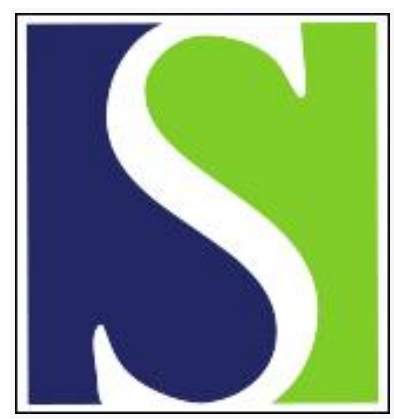

Scand J Work Environ Health 2018;44(4):370-376

https://doi.org/10.5271/sjweh.3723

Published online: 25 Mar 2018, Issue date: 01 Jul 2018

Is perception of safety climate a relevant predictor for occupational accidents? Prospective cohort study among blue-collar workers

by Ajslev JZN, Sundstrup E, Jakobsen MD, Kines P, Dyreborg J, Andersen LL

This study employs a five-item safety climate survey to investigate whether safety climate reports in 2012 were predictive for accident reports in 2014. It shows that a higher number of safety climate problems progressively increased the risk for accidents during the two-year follow-up. The five-item safety climate survey is a simple and important tool for risk assessment in blue-collar workplaces.

Affiliation: National Research Centre for the Working Environment, Lersø Parkallé 105, 2100 Copenhagen Ø, Denmark. jza@nrcwe.dk

Key terms: accident; blue-collar worker; occupational accident; occupational health and safety; occupational injury; predictor; prospective cohort; safety climate; safety culture

This article in PubMed: www.ncbi.nlm.nih.gov/pubmed/29574477

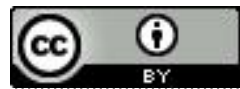




\title{
Is perception of safety climate a relevant predictor for occupational accidents? A prospective cohort study among blue-collar workers
}

\author{
by Jeppe Zielinski Nguyen Ajslev, PhD, ${ }^{1}$ Emil Sundstrup, PhD, ${ }^{1}$ Markus Due Jakobsen, PhD, ${ }^{1}$ Pete Kines, PhD, ${ }^{1}$ Johnny \\ Dyreborg, PhD, ${ }^{1}$ Lars Louis Andersen, PhD ${ }^{1}$
}

\begin{abstract}
Ajslev JZN, Sundstrup E, Jakobsen MD, Kines P, Dyreborg J, Andersen LL. Is perception of safety climate a relevant predictor for occupational accidents? A prospective cohort study among blue-collar workers. Scand J Work Environ Health. 2018:44(4):370-376. doi:10.5271/sjweh.3723
\end{abstract}

\begin{abstract}
Objectives The capability of safety climate to predict accidents has been the target of widespread debate in occupational health and safety research. The objective of this prospective cohort study was to employ a shortened five-item safety climate survey to investigate whether safety climate reports in 2012 are predictive for accidents reported within the last 12 months in 2014.
\end{abstract}

\begin{abstract}
Methods In both 2012 and 2014, 3864 blue-collar workers answered the Danish Working Environment and Health Cohort Study. Logistic regression was used to study the association [odds ratio (OR)] of reporting a work-related accident (yes/no) with more than one day of sickness absence (outcome) within the last 12 months in 2014 with the number of safety climate problems (predictor). The analyses were cumulatively adjusted for age, gender (model 1), socioeconomic class, occupational group, lifestyle (model 2), and previous accidents in 2012 (model 3).

Results Of the safety climate problems reported in 2012, 1017 (28\%) participants reported one problem, 357 $(10 \%)$ reported two and $614(17 \%)$ reported three or more problems. Using the number of safety climate problems as a continuous variable, all models showed a dose-response relationship between number of safety climate problems in 2012 and at least one accident in 2014 (trend-test, $\mathrm{P}<0.001$ ). Compared to participants with no safety climate problems, participants reporting two safety climate problems in 2012 had a higher risk for reporting an accident in 2014 [OR 1.84, 95\% confidence interval (CI) 1.22-2.77], and the risk was higher for participants reporting three or more safety problems (OR 2.22, 95\% CI 1.60-3.09).
\end{abstract}

Conclusions A higher number of safety climate problems progressively increased the OR for reporting at least one accident within the last 12 months at the two-year follow-up. The five-item safety climate survey is a simple and important tool that can be used as part of risk assessment in blue-collar workplaces.

Key terms occupational health and safety; occupational injury; safety culture

Safety at work is a cornerstone of good working places. An internationally acknowledged way of measuring safety at work is through the concept of "safety climate". Safety climate entered the field of occupational safety research with the landmark work of Dov Zohar (1). Over the last 35 years, the concept has developed as an approach for measuring the level of safety in organizations. Zohar specified safety climate as a particular aspect of organizational climate. It concerns managerial priorities towards safety, effects of safe conduct on organizational promotion, perceived status of safety officers/managers, perceived effects of safe conduct on social status in the organization, perceived effectiveness and importance of safety training, the risk level at the workplace and the perceived effectiveness of enforcement versus guidance in promoting safety. Safety climate perceptions refer to those attributes of policy, procedures and practices that indicate the priority of safety in an organization compared to other goals, ie, the priorities of competing goals as perceived by workers ("walk-the-talk" test) (1).

In later studies, safety climate has been reconceptualized in different directions. Varonen and Mattila (2) studied safety climate and its relationship to safety practice, safety of the work environment and occupational accidents in a cross-sectional study at Finnish wood-processing companies. They employed a Finnish reconceptualization of the safety climate concept based

1 National Research Centre for the Working Environment, Lersø Parkallé 105, 2100 Copenhagen Ø, Denmark.

Correspondence to: Jeppe Zielinski Nguyen Ajslev, National Research Centre for the Working Environment, Lersø Parkallé 105, 2100 Copenhagen Ø, Denmark. [E-mail: jza@nrcwe.dk] 
on Zohar's original work (3). Safety climate was measured using four factors: (i) organizational responsibility (ii) worker's safety attitudes (iii) safety supervision and (iv), company safety precautions. Their study showed that companies where worker ratings of "organizational responsibility" and "company safety precautions" were high, had a lower accident rate compared to companies where these ratings were low (2).

Whereas Zohar's initial questionnaire consisted of 40 items, in 2011 Kines et al (4) published their work on the Nordic Safety Climate Questionnaire (NOSACQ-50), where they presented a 7-factor, 50 -item tool for evaluating safety climate, based on psychological theory and earlier empirical studies (4). The questionnaire is suitable for research across a number of contexts, as the terminology is not specific to any sector or job task, but perhaps less suited for practical employment in organizational health and safety work. In 2017 Ajslev et al (5) showed that as few as five selected items from the NOSACQ-50, targeting managerial and employee commitment, participation and engagement, were able to show a progressively increasing risk-ratio for selfreported occupational accidents following the number of safety climate problems among a large population of workers across industrial sectors.

Numerous studies today explicitly or implicitly regard safety climate as a leading indicator capable of predicting safety outcomes $(5-10)$. However, research on safety climate as a leading indicator for accidents specifically is still limited, as most empirical studies are retrospective rather than prospective, as shown in a 2009 review by Payne et al (11). In addition, in 2006 Clarke identified a strong relationship between safety climate measurement and employee safety compliance and participation, but a weak relationship between safety climate and accidents (12). On the other hand, in 2000 Zohar (13) managed to show a statistically significant relationship between safety climate and "micro accidents" as a non-self-reported outcome for safety level in a five-month follow-up prospective study at a large manufacturing company. In a six-month prospective follow-up study in 2002, he showed that the leadershipbased model of safety climate was predictive for the number of minor injuries among 381 line workers at a metal processing plant (14).

In 1999 Hofmann \& Morgeson (15) showed that safety climate was significantly related to accidents among approximately 1200 manufacturing workers, in a cross-sectional case study based on 49 group leader's accident records. In a recent study however, Gilberg et al (16) found ambiguous results when investigating the longitudinal effects of safety climate on dropped-object accidents and hydrocarbon leaks in a survey study among 46100 employees in the Norwegian oil industry, as there were variations in safety climate measurements between different companies, and safety climate could only explain a limited part of this variation. Kongsvik et al (17) had previously discovered significant support for safety climate as a leading indicator for the same type of accidents in a study connecting survey data collected among 2188 off-shore workers to safety data from the Petroleum Safety Authorities in the same industry. As a result of this ambiguity in existing safety climate research, there is a need to further explore whether workers' perceptions of safety climate are a reliable predictor of future accidents. As indicated by, eg, the Zohar $(13,14)$ and Hoffmann \& Morgeson studies $(15)$, there is some evidence for this - yet these are based on relatively small populations. There is a need for studies among larger populations to help clarify whether safety climate could be used as an easy-to-use tool (walk-thetalk test), in proactive monitoring of companies' safety performance.

We designed a longitudinal study among a relatively large sample of blue-collar workers $(\mathrm{N}=3864)$ who responded to the Danish Working Environment and Health Cohort Study in both 2012 and 2014. In this prospective cohort study, we employ the same five items as earlier employed by Ajslev et al (5) to investigate whether reported safety climate issues in 2012 are predictive for reporting accidents within the last 12 months in 2014. If these questions are predictive for accidents up to two years later, there would be increased reason for organizations, researchers and health and safety professionals to employ safety climate in this shortened version as a quick way to attain a leading safety indicator in an organization.

\section{Methods}

\section{Study design}

This prospective cohort study uses questionnaire data from the Danish Working Environment and Health Cohort Study $(18,19)$. The reporting of this study conforms to the guideline "Strengthening the Reporting of Observational Studies in Epidemiology" (STROBE) in cohort studies (14). Following this we worked out a checklist in order to ensure that we reflected on the methodological and theoretical implications of the study.

\section{Participants}

The study population consisted of blue-collar workers who participated in both the 2012 (baseline) and 2014 round (follow-up) of the Danish Work Environment and Health Cohort Study $(18,19)$. In 2012, a representative 
random sample of 9961 blue-collar workers aged 18-64 years was extracted from Statistics Denmark and invited to participate, of these, 6249 were invited to participate again in 2014. In April 2012, these people received a letter with a link to the electronic survey; non-responders received two further notices (one by letter, one by phone) encouraging them to participate. Of the 6249 who were invited to participate in both rounds, 3864 participated in both 2012 and 2014. The procedure was the same in 2012 and 2014. Thus, the final sample for the present study consisted of 3864 workers. Baseline characteristics are shown in table 1.

\section{Ethical approval}

The study was notified to and registered by the Danish Data Protection Agency (Datatilsynet; journal number 2015-57-0074). According to Danish law, questionnaire and register-based studies need neither approval by ethical and scientific committee nor informed consent. All data were deidentified and analyzed anonymously.

\section{Safety climate}

The five items (see table 2) selected from NOSACQ-50 provide a short version covering vital aspects of the safety climate concept as agreed on from 1980 until $2011(1,4,13,20,21)$. The original NOSAQ-50 survey contains 50 items across 7 dimensions at both the managerial and worker level. As safety climate is only one of 51 topics in the Danish Work Environment and Health Cohort Study survey, only 5 items were allotted to safety climate for practical reasons. These particular questions were selected as they concern managerial level priorities of safety (question 1-3) and group level commitment to work safely (question 4-5), and thus are indicative of the true priority of safety. In order to enable comparisons between units (organizations), context-free statements were chosen (eg, management involves employees in decisions regarding safety), whereas context-specific statements (eg, are hard hats readily available at this site) were avoided. For each question, respondents replied on a 4-point scale of "strongly agree", "agree", "disagree", or "strongly disagree". All responses of disagree or strongly disagree were interpreted as a safety climate problem, except for question 5 (negated wording), where agree or strongly agree were interpreted as a safety climate problem. For further analyses, a variable containing information on the number of safety climate problems was generated ranging from 0 to $\geq 3$ problems.

\section{Work-related accidents}

To assess accidents, we asked the question: "Have you experienced $\geq 1$ work-related accident that resulted in $\geq 1$ days absence within the past 12 months?" with the response options ranging from 0 to $\geq 4$ accidents. A binary variable was generated with 0 representing no accidents, and 1 representing $\geq 1$ work-related accident.

\section{Covariates}

The analyses were adjusted for age (continuous), gender (male, female), socioeconomic class (explained below), occupational group (explained below), lifestyle (explained below) and previous accidents in 2012.

Socioeconomic class was coded into a binary variable according to Statistic Denmark's SOCIO13 variable: 1 representing employees engaged in work that requires basic level skills, and 2 representing other employees.

Occupational group was coded and categorized (1-9) according to the 1988 revision of the Danish version of the International Standard Classification of Occupations register (D-ISCO 08): (i) management work, (ii) work requiring knowledge at the highest level within the area concerned, (iii) work that requires medium level knowledge, (iv) regular office and customer service, (v) service and sales work, (vii) work in agriculture, forestry and fishing (excluding assisting), (vii) craftsmanship, (viii) operator and assembly work as well as transport work, and (ix) other manual work.

Lifestyle factors could potentially affect attention towards accidents and included smoking (never, exsmoker, yes) and body mass index (BMI, $\left.\mathrm{kg} / \mathrm{m}^{2}\right)$, which was determined from respondents' height and weight.

\section{Statistical analyses}

Logistic regression (PROC GENMOD, SAS version 9.4, SAS Institute, Cary, NC, USA) was used for modelling the odds ratio (OR) of work-related accidents during the two-year follow-up period (from 2012-2014). In the first set of analyses, we estimated the association between number of safety climate problems and workrelated accidents (yes/no). Analyses were adjusted for potential confounders: model 1 was adjusted for age and gender and model 2 was additionally adjusted for socioeconomic class, occupational group (DISCO), lifestyle (smoking habits and BMI), and previous accidents in 2012. In addition, we performed a sensitivity analysis (model 3, N=3565) to exclude workers who reported an accident in the year previous to 2012, as these may either be particularly accident prone due to risk behavior, may be increasingly aware of risks and therefore at reduced risk, or may be increasingly negative in relation to their safety climate. Results are reported as OR with $95 \%$ confidence intervals (CI).

In the second set of analyses, we explored the association between each of the five safety problem ques- 
Table 1. Participant characteristics at baseline. [SD=standard deviation; $D-I S C O=D a n i s h$ version of International Standard Classification of 0 ccupations]

\begin{tabular}{|c|c|c|c|c|c|c|c|c|}
\hline & \multicolumn{4}{|c|}{2012 population } & \multicolumn{4}{|c|}{ Final population } \\
\hline & $\mathrm{N}$ & Mean & SD & $\%$ & $\mathrm{~N}$ & Mean & SD & $\%$ \\
\hline \multicolumn{9}{|l|}{ Gender } \\
\hline Male & 5048 & & & 50.68 & 1945 & & & 50.34 \\
\hline Female & 4913 & & & 49.32 & 1919 & & & 49.66 \\
\hline Age & 9961 & 45.44 & 11.39 & & 3864 & 46.40 & 10.36 & \\
\hline Smoking & 2731 & & & 27.89 & 927 & & & 24.31 \\
\hline Ex-smoker & 2800 & & & 28.59 & 1123 & & & 29.45 \\
\hline No never & 4262 & & & 43.52 & 1763 & & & 46.24 \\
\hline Body mass index (kg/m²) & 9712 & 26.13 & 4.51 & & 3789 & 26.20 & 4.40 & \\
\hline \multicolumn{9}{|l|}{ Socioeconomic class } \\
\hline Work that requires basic level skills & 8159 & & & 81.91 & 3244 & & & 83.95 \\
\hline Other employees & 1802 & & & 18.09 & 620 & & & 16.05 \\
\hline \multicolumn{9}{|l|}{ D-ISCO 08} \\
\hline Without category & 86 & & & 0.86 & 42 & & & 1.09 \\
\hline Management work & 42 & & & 0.42 & 12 & & & 0.31 \\
\hline Work requiring knowledge at highest level in area concerned & 41 & & & 0.41 & 18 & & & 0.47 \\
\hline Work that requires medium level knowledge & 86 & & & 0.86 & 42 & & & 1.09 \\
\hline Regular office and customer service & 1583 & & & 15.89 & 764 & & & 19.77 \\
\hline Service and sales work & 3291 & & & 33.04 & 1271 & & & 32.89 \\
\hline Work in agriculture, forestry and fishing (excluding assisting) & 81 & & & 0.81 & 29 & & & 0.75 \\
\hline Craftsmanship & 1803 & & & 18.10 & 629 & & & 16.28 \\
\hline Operator and assembly work as well as transport work & 1173 & & & 11.78 & 435 & & & 11.26 \\
\hline Other manual work & 1774 & & & 17.81 & 622 & & & 16.10 \\
\hline \multicolumn{9}{|l|}{$\begin{array}{l}\geq 1 \text { accident leading to } \geq 1 \text { day of sickness absence in the } 12 \\
\text { months prior to completing the survey in } 2012\end{array}$} \\
\hline No & 8923 & & & 91.00 & 3529 & & & 92.19 \\
\hline Yes & 882 & & & 9.00 & 299 & & & 7.81 \\
\hline \multicolumn{9}{|l|}{$\begin{array}{l}\geq 1 \text { accident leading to } \geq 1 \text { day of sickness absence in the } 12 \\
\text { months prior to completing the survey in } 2014\end{array}$} \\
\hline No & & & & & 3550 & & & 91.87 \\
\hline Yes & & & & & 314 & & & 8.13 \\
\hline
\end{tabular}

tions and work-related accidents. The same models as described above were used. In addition, Spearman correlation coefficient was used to determine the correlation between each of the five safety problem questions.

\section{Results}

Table 1 shows baseline demographics, lifestyle, socioeconomic class, D-ISCO-categories and number of workers reporting accidents in the population of bluecollar workers in 2012 as well as the final population who replied to both the 2012 and 2014 rounds. Of the 3864 participants, 299 (7.8\%) reported an accident leading to one or more days of sickness absence in 2012. At follow-up in 2014, 314 (8.1\%) reported an accident within the past 12 months.

Table 2 shows the OR for reporting an accident in 2014 as a function of each safety climate question individually. In the fully adjusted model (model 2) questions 1 (OR 1.58, CI 1.19-2.11), 2 (OR 1.66; CI 1.28-2.17) and 3 (OR 1.62, CI 1.25-2.09) are shown to be particularly predictive of accidents two years later.

Table 3 shows the OR for reporting an accident in 2014 as a function of the number of safety climate problems reported in 2012. Of the participants, 1017 (28.46\%) reported one safety climate problem in 2012,
$357(9.99 \%)$ reported two problems, $308(8.62 \%)$ reported three, $248(6.94 \%)$ reported four, and 58 $(1.62 \%)$ reported five problems. In all models, a doseresponse relationship between the number of safety climate problems in 2012 and accidents in the 12 months prior to completing the questionnaire is shown. In the most moderate estimate, the fully adjusted analysis (model 2), participants reporting two safety climate problems had a higher risk for reporting an accident in the 2014 questionnaire (OR 1.84, CI 1.22-2.77) and the risk was higher for participants reporting three or more safety problems (OR 2.22, CI 1.60-3.09).

\section{Discussion}

The fully adjusted model reveals a dose-response relationship between reporting safety climate problems in 2012 and accidents in 2014. Compared to participants with no safety climate problems, those reporting two of the five safety climate problems included in the analysis had an $84 \%$ higher risk for reporting an accident, and workers reporting three or more had a $122 \%$ increased risk.

As such our prospective study supports earlier research pointing to the capability of safety climate questions to predict accidents $(1,13-15,17)$. At the 
Table 2. Odds ratio (OR) for reporting accidents in 2014 as a function of the number of each single safety climate problem in 2012. [Cl=confidence interval; D-ISCO=Danish version of International Standard Classification of Occupations].

\begin{tabular}{|c|c|c|c|}
\hline \multirow[t]{2}{*}{ Safety climate questions } & Model $1^{\mathrm{a}}$ & Model $2^{b}$ & Model $3^{c}$ \\
\hline & OR $(95 \% \mathrm{Cl})$ & OR $(95 \% \mathrm{Cl})$ & OR $(95 \% \mathrm{Cl})$ \\
\hline 1. "Management ensures that everyone receives the necessary information on safety" & $1.85(1.41-2.43)$ & $1.58(1.19-2.11)$ & $1.55(1.11-2.17)$ \\
\hline $\begin{array}{l}\text { 2. "Management encourages employees here to work in accordance with safety rules- } \\
\text { even when the work schedule is tight" }\end{array}$ & $1.77(1.38-2.28)$ & $1.66(1.28-2.17)$ & $1.64(1.21-2.22)$ \\
\hline 3. "Management involves employees in decisions regarding safety" & $1.70(1.33-2.16)$ & $1.62(1.25-2.09)$ & $1.65(1.23-2.21)$ \\
\hline 4. "We who work here help each other to work safely" & $1.36(1.04-1.87)$ & $1.33(0.98-1.82)$ & $1.64(1.16-2.31)$ \\
\hline $\begin{array}{l}\text { 5. "We who work here consider minor accidents as a normal part of our daily work" (ne- } \\
\text { gated or reversed item) }\end{array}$ & $1.44(1.12-1.85)$ & $1.26(0.97-1.64)$ & $1.27(0.94-1.72)$ \\
\hline
\end{tabular}

adjusted for age and gender.

${ }^{b}$ Model 1 + socioeconomic class, D-ISCO-group, lifestyle (smoking habits and body mass index) and previous accidents in 2012.

c Sensitivity analysis of Model 2, excluding those with previous accidents in 2012.

Table 3. Odds ratio (OR) for reporting accidents in 2014 as a function of the number of reported safety climate problems in 2012. [Cl=confidence interval; D-ISCO=Danish version of International Standard Classification of Occupations].

\begin{tabular}{|c|c|c|c|c|c|c|c|c|}
\hline \multirow{2}{*}{$\begin{array}{l}\text { Number of safety climate } \\
\text { problems }\end{array}$} & \multirow[t]{2}{*}{$\mathrm{N}$} & \multirow[t]{2}{*}{$\%$} & \multicolumn{2}{|c|}{ Model $1^{\text {a }}$} & \multicolumn{2}{|c|}{ Model $2^{b}$} & \multicolumn{2}{|c|}{ Model $3^{c}$} \\
\hline & & & OR & $95 \% \mathrm{Cl}$ & OR & $95 \% \mathrm{Cl}$ & OR & $95 \% \mathrm{Cl}$ \\
\hline 0 & 1586 & 44.38 & 1.00 & & 1.00 & & 1.00 & \\
\hline 1 & 1017 & 28.46 & 1.57 & $1.15-2.13$ & 1.35 & $0.98-1.87$ & 1.39 & $0.97-2.00$ \\
\hline 2 & 357 & 9.99 & 2.09 & $1.41-3.09$ & 1.84 & $1.22-2.77$ & 2.20 & $1.29-3.16$ \\
\hline$\geq 3$ & 614 & 17.18 & 2.66 & $1.94-3.64$ & 2.22 & $1.60-3.09$ & 2.26 & $1.55-3.27$ \\
\hline
\end{tabular}

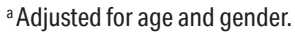

${ }^{\mathrm{b}}$ Model 1 + socioeconomic class, D-ISCO-group, lifestyle (smoking habits and body mass index) and previous accidents in 2012.

c Sensitivity analysis of Model 2, excluding those with previous accidents in 2012.

same time, the study shows that the five-item survey could be a practical (quick) and easy-to-use, alternative to the NOSACQ-50 questionnaire if the aim is to provide a leading indicator of whether an organization or a company are in particular risk for suffering future accidents.

The study also shows that safety climate indicators have a predictive capacity in relation to accidents, not only at the group or organizational level, as employed by Zohar and those inspired by his work $(6,7,9,21)$, but also at a population level, where each respondent's answers represent five safety climate dimensions of a group or organization. Our results in particular relate to employees engaged in work that requires basic-level skills and likely others as well. Still, it is doubtful whether safety climate would also be predictive of accidents among low-risk job groups such as employees engaged in sedentary work, office workers or managers.

In our results section, we place main emphasis on model 2, as this is the fully adjusted model including all selected participants. We performed the model 3 or sensitivity analysis to exclude workers who reported an accident in the 12 months previous to 2012, as such individuals may be increasingly aware of risks and therefore at reduced risk or may be increasingly negative in relation to their safety climate. This could mean that participants who reported an accident in 2012 would also be more likely to report a negative safety climate as a result of their accident. Therefore the sensitivity analysis excludes these. In general, we would have thought this analysis would lead to more cautious estimates in model 2. This was somewhat the case. In a numerical sense the fully adjusted model 2 in table 3 shows lower OR than model 3. However, the only significant difference between models 2 and 3 is in table 2, where model 3 shows four out of five items to be significantly associated with accidents, whereas model 2 only displays significance for three out of the five items. This is likely because workers or workplaces where respondents reported an accident in 2012, are more aware of safety and may have taken preventive actions, and therefore were better at avoiding accidents.

Table 2 shows our explorative analysis of the single items on the five-item safety climate survey. In model 2 , the three questions on managerial engagement in safety are the ones that are singularly relevant predictors for future reported accidents. That managerial engagement is particularly relevant is consistent with earlier results on both health and safety $(22,23)$ and the risk for musculoskeletal disorders (24) in the construction industry. The difference between model 2 and 3 (ie, sensitivity analysis) may be due to workers who have not experienced an accident and who are not so engaged in their own and their coworker's safety, compared to workers (and workplaces) who experienced an accident, have learned from past accidents, and thus engage in pre- 
venting reoccurrences (25). In the latter case, accident prevention seems to be more independent of whether their colleagues help them to work safely, while they are still dependent on management involvement.

The results displayed in table 2 may point in the direction that even further reduction of the short version of the safety climate questionnaire could be justified. However, an earlier study shows that there is a progressively increasing risk for reporting accidents, even above three safety climate problems (5). Therefore we would not immediately recommend such a reduction. However, given the frequent limited number of questions allowed in surveys on different topics, there could be reasons for only employing questions 1, 2 and 3 .

\section{Limitations and strengths}

It is a strength of this study that the prospective design enables inferences about the relationship between safety climate reports in 2012 and accidents as an outcome in the 12 months prior to completing the survey in 2014 . However, a limitation is that the study is based on selfreports rather than medical or company sickness absence registration data. Hence, the assessment of safety climate and accidents may be affected by recall bias along with current health status and working environment factors. Recall bias is a limitation, even though an accident might be seen as a standalone traumatic event, which is easier to recall later than other work-related events. It is a limitation that the survey asks for accidents within the last 12 months, while our follow-up is after two years in 2014. Of the 6249 blue collar-workers who were invited to participate in both 2012 and 2014, 3864 replied to the questionnaire in 2014. Hence, we cannot rule out that some workers could have experienced long term absence from work due to an accident during 2013, which could be a reason for not responding to the questionnaire in 2014. This scenario could have implications for the validity of the present results, with a possibility of underestimating the true association between safety climate complaints and accidents. Hence, our results seem to be conservative in this regard. In addition, extending the recall period to two years could be at the expense of an increased recall bias. Another limitation is that we did not account for change of jobs during 2012 and 2014. Although only a small proportion of the blue-collar workers may have changed to completely other types of jobs such as office work, some may have changed workplace during the follow-up period. In such case, the prospective association between current perception of safety climate and the risk for future accidents may be weakened, and the estimates of the present study more conservative. In questionnaire studies with voluntary participation, there may also be selection bias. However, considering that the 2012 study sample was very similar to the final sample size - consisting of those replying both in 2012 and 2014 - there does not seem to be any particular selection bias.

The common methods bias could have affected the validity of the present results, and also the causal relation between safety climate measures and reported accidents. Future studies should focus on this particular bias in order to improve our knowledge of the predictive validity of safety climate as a proactive indicator for accidents at work.

With 3864 participants, the study's size strengthens the generalizability of the findings. The study is, however, limited to including workers in Statistic Denmark's SOCIO13 variables 1 and 2, representing "employees engaged in work that requires basic level skills", and "other employees". This means that we most likely only include workers who are engaged in a wide variety of both manual and sedentary work. In total, 3721(96.3\%) of our participants are from the D-ISCO groups: regular office and customer service, service and sales work, craftsmanship, operator and assembly work as well as transport work, and other manual work. As such, our results are highly generalizable for workers in these job groups, but not for managers and knowledge workers.

\section{Concluding remarks}

The five-item safety climate survey is capable of predicting reported accidents among blue-collar workers in the two-year follow-up. A higher number (two or more) of safety climate problems reported in 2012 progressively increased the risk for reporting an accident in the 12 months prior to completing the survey in 2014. In the single-item questionnaire analysis, questions regarding managerial engagement (questions 1-3) seem to be most relevant as single factors for predicting accidents. The study supports the usage of the five-item safety climate survey as a reasonable way of evaluating the risk for accidents.

\section{Acknowledgements}

The study was carried out at the expense of the National Research Centre for the Working Environment in Denmark. Jeppe Ajslev and Lars Andersen designed the study, Jeppe Ajslev and Emil Sundstrup wrote the initial draft for the article, Lars Andersen performed the statistical analysis, Pete Kines, Johnny Dyreborg and Markus Jakobsen contributed with feedback about study design. All authors contributed with comments and revisions of the final manuscript.

The authors declare no conflict of interests. 


\section{References}

1. Zohar D. Safety climate in industrial organizations: theoretical and applied implications. J Appl Psychol 1980 Feb;65(1):96-102. http://dx.doi.org/10.1037/00219010.65.1.96.

2. Varonen U, Mattila M. The safety climate and its relationship to safety practices, safety of the work environment and occupational accidents in eight wood-processing companies. Accid Anal Prev 2000 Nov;32(6):761-9. http://dx.doi. org/10.1016/S0001-4575(99)00129-3.

3. Seppälä A. Evaluation of safety measures, Their Improvement and Connections to Occupational Accidents. Helsinki: People and Work. Finnish Institute of Occupational Health; 1992. Report No. 1/92.

4. Kines P, Lappalainen J, Mikkelsen KL, Olsen E, Pousette A, Tharaldsen J et al. Nordic Safety Climate Questionnaire (NOSACQ-50): A new tool for diagnosing occupational safety climate. Int J Ind Ergon 2011 Nov;41(6):634-46. http://dx.doi.org/10.1016/j.ergon.2011.08.004.

5. Ajslev J, Dastjerdi EL, Dyreborg J, Kines P, Jeschke KC, Sundstrup E et al. Safety climate and accidents at work: cross-sectional study among 15,000 workers of the general working population. Saf Sci 2017 Jan;91:320-5. http:// dx.doi.org/10.1016/j.ssci.2016.08.029.

6. Chen Y, McCabe B, Hyatt D. Impact of individual resilience and safety climate on safety performance and psychological stress of construction workers: A case study of the Ontario construction industry. J Safety Res 2017 Jun;61:167-76. http://dx.doi.org/10.1016/j.jsr.2017.02.014.

7. Kvalheim SA, Dahl Ø. Safety compliance and safety climate: A repeated cross-sectional study in the oil and gas industry. J Safety Res 2016 Dec;59:33-41. http://dx.doi. org/10.1016/j.jsr.2016.10.006.

8. Kvalheim SA, Antonsen S, Haugen S. Safety climate as an indicator for major accident risk: can we use safety climate as an indicator on the plant level? Int J Disaster Risk Reduct 2016 Sep;18:23-31. http://dx.doi.org/10.1016/j. ijdrr.2016.05.011.

9. Pousette A, Larsman P, Eklöf M, Törner M. The relationship between patient safety climate and occupational safety climate in healthcare - A multi-level investigation. J Safety Res 2017 Jun;61:187-98. http://dx.doi.org/10.1016/j. jsr.2017.02.020.

10. Zahoor H, Chan AP, Utama WP, Gao R. A Research Framework for Investigating the Relationship between Safety Climate and Safety Performance in the Construction of Multistorey Buildings in Pakistan. Procedia Eng 2015 Jan;118:5819. http://dx.doi.org/10.1016/j.proeng.2015.08.488.

11. Payne SC, Bergman ME, Beus JM, Rodríguez JM, Henning JB. Safety climate: leading or lagging indicator of safety outcomes? J Loss Prev Process Ind 2009 Nov;22(6):735-9. http://dx.doi.org/10.1016/j.jlp.2009.07.017.

12. Clarke S. The relationship between safety climate and safety performance: a meta-analytic review. J Occup Health Psychol 2006 Oct;11(4):315-27. http://dx.doi. org/10.1037/1076-8998.11.4.315.

13. Zohar D. A group-level model of safety climate: testing the effect of group climate on microaccidents in manufacturing jobs. J Appl Psychol 2000 Aug;85(4):587-96. http://dx.doi. org/10.1037/0021-9010.85.4.587.

14. Zohar D. The effects of leadership dimensions, safety climate, and assigned priorities on minor injuries in work groups. J Organ Behav 2002 Feb;23(1):75-92. http://dx.doi. org/10.1002/job.130.

15. Hofmann DA, Morgeson FP. Safety-related Behavior as a Social Exchange: The Role og Perceived Organizational Support and Leader-Member Exchange. J Appl Psychol 1999;84(2):286-96. http://dx.doi.org/10.1037/00219010.84.2.286

16. Gilberg A, Ekle R, Bye RJ, Kongsvik T. Why Measure Safety Climate? A longitudinal study of the relationship between safety climate measurements and safety performance. Saf Reliab Complex Eng. Syst. CRC Press; 2015. p. 3771-9.

17. Kongsvik T, Kjøs Johnsen SÅ, Sklet S. Safety climate and hydrocarbon leaks: an empirical contribution to the leading-lagging indicator discussion. J Loss Prev Process Ind 2011 Jul;24(4):405-11. http://dx.doi.org/10.1016/j. jlp.2011.02.004.

18. NRCWE. Arbejdsmiljø og Helbred 2012 [Work environment and health in Denmark 2012]. Copenhagen, Denmark: National Research Centre for the Working Environment; 2012.

19. NRCWE. Danskernes Arbejdsmiljø 2014 [Work environment and health in Denmark 2014]. Copenhagen, Denmark: National Research Centre for the Working Environment; 2014 p150.

20. Neal A, Griffin MA. Safety climate and safety behaviour. Aust J Manag 2002;27(1_ suppl):67-75. http://dx.doi. org/10.1177/031289620202701S08.

21. Zohar D. Thirty years of safety climate research: reflections and future directions. Accid Anal Prev 2010 Sep;42(5):151722. http://dx.doi.org/10.1016/j.aap.2009.12.019.

22. Choudhry RM, Fang D, Mohamed S. The nature of safety culture: A survey of the state-of-the-art. Saf Sci 2007 Dec;45(10):993-1012. http://dx.doi.org/10.1016/j. ssci.2006.09.003.

23. Kines P, Andersen LP, Spangenberg S, Mikkelsen KL, Dyreborg J, Zohar D. Improving construction site safety through leader-based verbal safety communication. J Safety Res 2010 Oct;41(5):399-406. http://dx.doi.org/10.1016/j. jsr.2010.06.005.

24. Ajslev JZ, Persson R, Andersen LL. Contradictory individualized self-blaming: a cross-sectional study of associations between expectations to managers, coworkers, one-self and risk factors for musculoskeletal disorders among construction workers. BMC Musculoskelet Disord 2017 Jan;18(1):13. http://dx.doi.org/10.1186/s12891-016-1368-1.

25. Kjellen U. Prevention of Accidents Through Experience Feedback. 1 edition. London; New York: CRC Press; 2000.

Received for publication: 19 September 2017 